\title{
Enhanced 2-phenylethanol production by newly isolated
}

\author{
Fengxue Xin ${ }^{1}$, Wei Yan $^{2}$, Xiujuan Qian ${ }^{2}$, Wenming Zhang ${ }^{1}$, Jie Zhou ${ }^{2}$, Weiliang Dong ${ }^{1}$, \\ Bin $\mathrm{Xu}^{2}$, and Min Jiang ${ }^{1}$ \\ ${ }^{1}$ Nanjing University of Technology \\ ${ }^{2}$ Nanjing Tech University
}

April 28, 2020

\begin{abstract}
A unique Meyerozyma sp. strain YLG18 was obtained in this study, which was capable of producing 2-phenylethanol through both Ehrlich and Shikimate pathways. Response surface methodology (RSM) was implemented to improve the maximum 2-PE production. At optimized conditions: temperature, 24.7; initial glucose, $63.54 \mathrm{~g} / \mathrm{L}$; initial L-phe, $10.70 \mathrm{~g} / \mathrm{L}, 2-\mathrm{PE}$ production was increased to $2.55 \mathrm{~g} / \mathrm{L}$, leading to $104 \%$ increase compared to the pre-optimized one. In situ product recovery (ISPR) could further help improve the final 2-PE production to $3.20 \mathrm{~g} / \mathrm{L}$ with fatty acid methyl ester as the extractant, representing the highest 2-PE production by using Meyerozyma sp.. Furthermore, genes involved in 2-PE synthesis were identified and their expression levels between Shikimate pathway and Ehrlich pathway were compared. Based on the genomic and transcriptional analysis, a penta-functional enzyme AroM in Shikimate pathway and an aspartate aminotransferase (AAT) with the potential to convert phenylalanine into phenylpyruvate in Ehrlich pathway were identified. These findings would help broaden our knowledge and add to the pool of known 2-PE generating microbes and genes.
\end{abstract}

\section{1、Introduction}

2-Phenylethanol (2-PE) is an aromatic alcohol, which can be naturally found in essential oils of many plants, such as hyacinths, jasmine and lilies. 2-PE has been widely used in cosmetics, perfume and food industries owning to its delicate rose scent (Scognamiglio, Jones, Letizia, \& Api, 2012). Furthermore, 2$\mathrm{PE}$ is also an important raw material for the derivatives synthesis, among which phenylethyl acetate is a valuable fragrance compound, and p-hydroxyphenylethanol is widely used in pharmaceutical and fine chemicals industries (Masuo, Osada, Zhou, Fujita, \& Takaya, 2015; Yin et al., 2015). Although 2-PE can be extracted from flowers and plants, however, the extremely low concentration hinders it application (Feng et al., 2015). Alternatively, 2-PE can be chemically synthesized, however, its quality is greatly affected because of the harsh condition and toxic reagents used (Etschmann, Bluemke, Sell, \& Schrader, 2002). Currently, the price of 2-PE produced through natural routes, such as extraction from rose petals or bio-converted from renewable resources is approximately USD $1,000 / \mathrm{kg}$. However, it is only USD $5.0 / \mathrm{kg}$ for chemically synthesized 2-PE from benzene and styrene (Hua \& Xu, 2011). Therefore, bio-synthesis of 2-PE has become an appealing option owning to its environmentally friendly property and mild conditions.

In nature, many wild-type microorganisms have been identified and characterized to be capable of producing 2-PE, most of which are from eukaryotes, including Saccharomyces sp., Kluyveromyces marxianus, Yarrowia lipolytica, Aspergillus oryzae, and Pichia sp., etc (Huang, Lee, \& Chou, 2001; Masuo et al., 2015). In addition, some prokaryotes have also been reported to produce 2-PE, such as Microbacterium foliorum, Proteus vulgaris, and Psychrobacter sp., 2-PE can be either converted from L-phenylalanine (L-phe) through three steps catalysis of Ehrlich pathway or produced from glucose through Shikimate pathway. As multiple steps are needed for 2-PE production through Shikimate pathway, lower concentration usually occurred when 2-PE was directly synthesized from glucose. Accordingly, Ehrlich pathway is thought to be more promising 
for 2-PE production. It should be noticed that the lipophilic 2-PE could make the lipid membrane structure a preferential binding target, resulting in the collapse of transmembrane gradients and consequently the loss of cell viability (Sikkema, de Bont, \& Poolman, 1995), hence, strain development including mutation, selection, or genetic modification has been comprehensively adopted to improve the final 2-PE production. For instance, the newly isolated K. marxianus CCT 7735 could generate $3.44 \mathrm{~g} / \mathrm{L}$ of 2-PE through Ehrlich pathway under optimized conditions (Azevedo, Santos, Vieira, Gomes, \& Batista, 2018). S. cerevisiae BY4741, which overexpressed ARO10 and contained an aro8 $\Delta$ deletion could produce $96 \mathrm{mg} / \mathrm{L}$ of 2-PE from glucose (Shen, Nishimura, Matsuda, Ishii, \& Kondo, 2016). S. cerevisiae SPO810, in which ARO8 and ARO10 were co-expressed could finally produce $2.61 \mathrm{~g} / \mathrm{L}$ of 2 -PE with fed batch fermentation (Yin et al., 2015).

In the present study, novel 2-PE-generating microbes were first isolated and characterized. To further improve 2-PE production, statistical design of experimental strategy and in situ extraction strategy were used to improve final 2-PE titer. Transcriptome analysis on genes involved in 2-PE synthesis were also identified and characterized.

\section{2、Material and methods}

\subsection{Isolation and molecular identification of strain YLG18}

Soil samples from Xuanwu Lake, Nanjing, China were used as inocula in order to screen bacteria capable of producing 2-PE. The soil samples were added into flasks containing $50 \mathrm{~mL}$ of defined mineral salts medium (MMT) spiked with $40 \mathrm{~g} / \mathrm{L}$ of glucose as the carbon source. After $96 \mathrm{~h}$ incubation at $30{ }^{\circ} \mathrm{C}$ and a shaking speed of $200 \mathrm{rpm}, 0.5 \mathrm{~mL}$ culture was spread on a Petri dish containing MMT medium, which was conducted in aseptic environment. Then different colonies were picked into mineral salts medium using L-phe as the sole nitrogen source for detection of 2-PE production capabilities. Eventually, a 2-PE-producing bacterium named YLG18 was obtained. Unless stated otherwise, the strain was grown in mineral salts medium with L-phe as the sole nitrogen source at $30{ }^{\circ} \mathrm{C}$.

The defined mineral salts medium contained (per liter of distilled water): glucose $40 \mathrm{~g}$, L-phe $5 \mathrm{~g}$, NaCl 1.0 g, $\mathrm{MgCl} 2 \cdot 6 \mathrm{H} 2 \mathrm{O} 0.5 \mathrm{~g}, \mathrm{KH} 2 \mathrm{PO} 40.2 \mathrm{~g}, \mathrm{KCl} 0.3 \mathrm{~g}, \mathrm{CaCl} 2 \cdot 2 \mathrm{H} 2 \mathrm{O} 0.015 \mathrm{~g}$, TES $2.292 \mathrm{~g}$, uracil $0.1 \mathrm{~g}$, thiamine $3 \mathrm{mg}$. In addition, $1 \mathrm{~mL}$ of trace element solution and $10 \mathrm{~mL}$ of salts solution were added to $1 \mathrm{~L}$ medium. Then the medium was dispended into triangle shaker, which were wrapped with gauze, autoclaved for 20 minutes and cooled down to room temperature.

Genomic DNA of the cultures was extracted and purified with DNeasy tissue kit (Qiagen, Germany) according to manufacturer's instructions. The genomic DNA was used as a template for PCR amplification of the 18S rRNA gene with a pair of universal fungi primer ITS1 (5'-CCGTAGGTGAACCTGCGG-3') and ITS4 (5'TCCTCCGCTTATTGATATGC-3'). The obtained PCR products were purified with a PCR purification kit (Qiagen, Germany) and sequenced using an ABI DNA sequencer (Applied Biosystems, USA). The 18S rRNA gene sequence was aligned using the BLAST algorithm and was deposited in the GenBank database with an accession number of WHVZ00000000.

\subsection{Optimization of fermentation conditions}

All batch fermentation studies were carried out in $250 \mathrm{~mL}$ triangle shakers containing $50 \mathrm{~mL}$ of mineral salts medium with initial $\mathrm{pH}$ of 5.8. The medium-filled triangle shakers were wrapped with gauze and aluminum foil before being autoclaved. L-phe was first filter-sterilized and then added into the medium as the sole nitrogen source. Inoculation was carried out by adding $2 \%$ of seed culture (strain YLG18) to the medium. The bottles were incubated at $30 \operatorname{deg} \mathrm{C}$ and $200 \mathrm{rpm}$ in a shaking incubator for $96 \mathrm{~h}$. Detailed optimization studies regarding the production of 2-PE were carried out by using the response surface methodology (RSM). Three factors (temperature, initial glucose concentration, and initial L-phe concentration) were chosen as independent variables, while 2-PE concentration was the dependent variable. An experimental strategy based 
on central composite design (CCD) was obtained using Design Expert version 7.0 (Stat-Ease, Minneapolis, USA). The values of the response variable obtained were fitted to a second-order polynomial equation:

$\mathrm{Yi}=\beta 0+[?] \beta \mathrm{i} \mathrm{xi}+[?] \beta \mathrm{iixi2}+[?] \beta \mathrm{ijxixj}$

where $\mathrm{Yi}$ is the predicted response, $\mathrm{xi}$, xj are independent variables, which influence the dependent variable $\mathrm{Y} ; \beta 0$ is the offset term; $\beta \mathrm{i}$ is the ith linear coefficient; $\beta \mathrm{ii}$ is the ith quadratic coefficient and $\beta \mathrm{ij}$ is the ijth interaction coefficient. Statistical analysis of the model was performed by using analysis of variance (ANOVA) in a statistical software package.

Subsequently, the optimized condition obtained from RSM was applied to batch fermentation in a 3.0-L bioreactor (BIOSTATR B plus, Sartorius, Germany). The bioreactor was filled with $2.0 \mathrm{~L}$ of mineral salts medium and operated at $30{ }^{\circ} \mathrm{C}$ with an agitation rate of $200 \mathrm{rpm}$.

\subsection{Analytic method}

Fermentation broth samples were analyzed for biomass growth, glucose and L-phe utilization, and 2-PE production. Biomass was determined by measuring optical density at $600 \mathrm{~nm}$ with appropriate dilution using a UV-visible spectrophotometer (Lambda-25, Perkin-Elmer, USA). Glucose was analyzed by a 1260 Series HPLC system (Agilent Technologies Inc.) equipped with an Aminex HPX-87H column (BioRad, Richmond, CA, USA) and a Refractive Index Detector (RID). Samples were run at $75{ }^{\circ} \mathrm{C}$ with $0.6 \mathrm{~mL} / \mathrm{min}$ eluent of $5 \mathrm{mM}$ sulfuric acid. Substrate (L-phe) and product (2-PE) were measured by a 1260 Series HPLC system (Agilent Technologies Inc.) equipped with an AcclaimTM 120-C18 column (Thermo Scientific, China) and UV-detector at $210 \mathrm{~nm}$. 50\% sterile water and $50 \%$ (v/v) methanol were pumped isocratically through Agilent 1260 quat pump at $0.6 \mathrm{~mL} / \mathrm{min}$. The column was kept at temperature of 30. Five-point standard curves were obtained by running standard solutions containing L-phe and 2-PE.

\subsection{In situ product removal (ISPR) for 2-PE production}

Extractive bioconversion was carried out with the addition of different organic solvents as extractants. These extractants were added directly with an aqueous/organic ration of $1: 1(\mathrm{v} / \mathrm{v})$ when the bioconversion started. Controls were conducted without addition of any solvent. The bioconversion was carried out at $180 \mathrm{rpm}, 30$ $\mathrm{oC}$ in 500-mL flask. At the end of the bioconversion, samples were collected and 2-PE concentration in both organic and aqueous phases was measured (Stark, 2003).

\section{5. qRT-PCR-based validation and analysis}

qRT-PCR was conducted to verify the expression of genes potentially involved in the biosynthesis pathway. Total RNA was extracted from strain YLG18 cultured after $48 \mathrm{~h}$ using the FastPure Cell/Tissue Total RNA Isolation Kit (Vazyme, Nanjing, China) and reverse-transcribed into cDNA using the HiScript II Q RT SuperMix for qPCR (+gDNA wiper) (Vazyme, Nanjing, China). PCR primers were designed with SnapGene 1.1.3 based on the nucleotide sequence of reference and antioxidant-related genes from the RNA-Seq data (Table 1). qRT-PCR was performed using ChammQTM SYBR qPCR Master Mix (High ROX Premixed) (Vazyme, Nanjing, China) and an ABI StepOnePlus (Applied Biosystems). qRT-PCR was performed with the follow thermocycling parameters: $95 \operatorname{degC}$ for $30 \mathrm{~s}$, followed by 40 cycles of $95 \operatorname{deg} \mathrm{C}$ for $10 \mathrm{~s}$ and 55 $\operatorname{deg} \mathrm{C}$ for $30 \mathrm{~s}$. Triplicate analyses of each cDNA sample were performed, and the relative expression levels of genes in each group were normalized to $18 \mathrm{SrRNA}$ expression. The 2- $\Delta \Delta \mathrm{Ct}$ comparative threshold cycle (Ct) method was used to evaluate the relative expression levels of target genes (Livak \& Schmittgen, 2000). The values reported represent the average of 3 biological replicates (Perdiguero et al., 2012).

\section{3、Results and discussion}

\subsection{Isolation and phylogenetic analysis of Meyerozymasp. strain YLG18}

In this study, L-phe was used as the sole nitrogen source for the isolation of 2-PE generating strains (Fig. 1A). After more than 5 consecutive transfers in the medium spiked with $5 \mathrm{~g} / \mathrm{L}$ of L-phe and $40 \mathrm{~g} / \mathrm{L}$ of glucose, one colony named YLG18 gave the highest 2-PE production and molar conversion. Furthermore, 
YLG18 can even produce $100 \mathrm{mg} / \mathrm{L}$ of 2-PE in synthetic medium without supplementation of L-phe via the de novo pathway (Fig. 1B), which surpassed that using metabolically engineered S. cerevisiae (96 mg/L) via de novo pathway (Shen et al., 2016). The $18 \mathrm{~S}$ rDNA genes amplified from the genomic DNA of culture YLG18 showed $99 \%$ identity to Meyerozyma guilliermondii when blasted with the bacterial sequences in the GenBank database. Thus, this culture is designated as Meyerozyma sp. strain YLG18 (Table 2). $M$. guilliermondii is known to be an ascomycetous yeast, which is broadly used for the production of riboflavin, xylitol and industrial enzymes (Papon et al., 2013). Actually, M. guilliermondii has been reported to produce 2-PE through biological conversion of L-phe, and the highest 2-PE production could reach $1.61 \mathrm{~g} / \mathrm{L}$ (Karolina, Katarzyna, Daria, \& Jolanta, 2017), however, the underlying mechanism for 2-PE production has not been clearly elaborated. Therefore, this newly isolated wild-type 2-PE-producing Meyerozyma sp. strain YLG18 may further broaden our knowledge and add to the pool of known 2-PE generating microbes.

2-PE can increase the cell membrane fluidity and reduce the uptake of amino acids and glucose, leading to the low microbial growth and 2-PE production. The increased permeability of membrane would accelerate the transmembrane diffusion of ions and small molecular metabolites, and disrupt the transmembrane proton potential (Seward, Willetts, Dinsdale, \& Lloyd, 1996). Studies have reported that $2.0 \mathrm{~g} / \mathrm{L}$ of 2-PE could completely inhibit the growth of S. cerevisiae W303-1A andS. cerevisiae Giv 2009 (Stark, Münch, Sonnleitner, Marison, \& Stockar, 2002). Therefore, to further determine the 2-PE tolerance potential of strain YLG18, the 2-PE tolerance of strain YLG18 was investigated. Different concentrations of exogenous 2-PE ranging from $1.5 \mathrm{~g} / \mathrm{L}$ to $4.0 \mathrm{~g} / \mathrm{L}$ were added into the fermentation medium initially. As seen from Fig. 1C, with the increase of exogenously added 2-PE, the inhibition effect on strain growth was obviously observed. For instance, with the supplementation of $3.5 \mathrm{~g} / \mathrm{L}$ of 2-PE, OD600 reached 10, which is only half of that in the presence of $1.5 \mathrm{~g} / \mathrm{L} 2-\mathrm{PE}$. When the concentration of exogenous 2-PE reached $4.0 \mathrm{~g} / \mathrm{L}$, strain growth was almost completely inhibited. Nevertheless, the higher 2-PE tolerance level of strain YLG18 compared to current reported 2-PE producers indicated that it may be promising candidate for high 2-PE production (Schrader, Etschmann, Sell, Hilmer, \& Rabenhorst, 2004).

\subsection{Determination of influencing factors for 2-PE production byMeyerozyma sp. strain YLG18}

To further improve the final 2-PE titer, various strategies including fermentation condition optimization and process integration have been developed. During fermentation process, carbon source has been proved as an important factor influencing cell growth, L-phe consumption and 2-PE molar conversion. Therefore, different carbon sources including glycerol, glucose, xylose, rapeseed oil and NaAc were chosen for 2-PE production (Fig 2A). After $96 \mathrm{~h}$ fermentation in mineral salts medium, the highest $1.25 \mathrm{~g} / \mathrm{L}$ of 2-PE with glucose as the carbon source occurred with $51.3 \%$ molar conversion, which is $38.89 \%, 56.25 \%, 316.67 \%$ and $150 \%$ higher than that using xylose, glycerol, rapeseed oil and NaAc as substrate, respectively. It should be noticed that although glycerol is more reduced than glucose and provide more NADH, which is critical for the last reduction step in Ehrlich pathway, it only gave $0.8 \mathrm{~g} / \mathrm{L}$ of 2-PE. Also, previous studies have shown that lower $\mathrm{pH}$ is relatively favorable for cell growth, but unfavorable for 2-PE production $(\mathrm{Mu}, \mathrm{Hu}$, $\mathrm{Liu}, \mathrm{Zhao}, \& \mathrm{Xu}, 2014)$. However, when sodium acetate was used as carbon source to increase $\mathrm{pH}, 2 \mathrm{PE}$ production was only $0.5 \mathrm{~g} / \mathrm{L}$.

The effect of temperature ranging from 25 to $37^{\circ} \mathrm{C}$ was also evaluated in synthetic medium containing $30.0 \mathrm{~g} / \mathrm{L}$ of glucose (Fig. 2B). The highest $1.55 \mathrm{~g} / \mathrm{L}$ of $2-\mathrm{PE}$ was obtained at $30^{\circ} \mathrm{C}$ with molar conversion of $70.86 \%$. Studies have shown M. guilliermondii preferred $37^{\circ} \mathrm{C}$ for ethanol production, however, slightly lower 2-PE production of $1.3 \mathrm{~g} / \mathrm{L}$ occurred, and an OD600 of 14.32 was achieved at $37^{\circ} \mathrm{C}$, indicating that elevated temperatures unfavored 2-PE production and microbial growth for strain YLG 18. Similar results were also obtained when other M. guilliermondii strain, such as M. guilliermondii WUT22 was used for bioconversion of L-phe to 2-PE (Diniz, Rodrigues, Fietto, Passos, \& Silveira, 2013; Ferreira et al., 2015).

During the bioconversion process, $30 \mathrm{~g} / \mathrm{L}$ of glucose can be rapidly consumed during $48 \mathrm{~h}$, however, 3.65 $\mathrm{g} / \mathrm{L}$ of L-phe was still left over with initial concentration of $5 \mathrm{~g} / \mathrm{L}$ (data not shown). Hence, different initial glucose concentrations were assessed for their effects on 2-PE production. As seen in Fig. 2C, when initial glucose concentration was $40.0 \mathrm{~g} / \mathrm{L}$, the highest $2.17 \mathrm{~g} / \mathrm{L}$ of $2-\mathrm{PE}$ and $77.6 \%$ of molar conversion were 
achieved, respectively. The molar conversion would decrease with the increase of glucose concentration. When glucose concentration was above $40 \mathrm{~g} / \mathrm{L}$, OD600 dropped from 38.15 to 31.96 with the increase of glucose concentration. Especially, 2-PE production kinetics is paralleled to that of microbial growth, suggesting that 2-PE production by strain YLG18 was closely related to cell growth.

As L-phe was used as the nitrogen source for both microbial growth and 2-PE production, different amounts of L-phe were also evaluated for the improvement of 2-PE production. It can be seen from Fig. 2D that 2-PE production kinetics was basically consistent with the yeast growth. When L-phe concentration was $7 \mathrm{~g} / \mathrm{L}, 2.22$ $\mathrm{g} / \mathrm{L}$ of 2-PE and a maximum OD600 of 35.72 were achieved, which was higher than that using $S$. cerevisiae Ye9- 612 E (0.85 g/L) (Eshkol, Sendovski, Bahalul, Kashi, \& Fishman, 2015; Stark et al., 2003). However, the molar conversion with $7.0 \mathrm{~g} / \mathrm{L}$ of $2-\mathrm{PE}$ was only $79 \%$, which could be because the molar conversion was negatively correlated with the initial L-phe concentration. This finding proved that high L-phe concentration in a certain range is beneficial for 2-PE production, but would reduce the conversion yield, which was also verified by other study, in which L-phe concentrations above $4.0 \mathrm{~g} / \mathrm{L}$ did not lead to the increase of 2-PE titer by using K. marxianus CCT 7735 (Azevedo et al., 2018).

\subsection{Optimization of fermentation conditions for enhanced 2-PE production from L-phe}

Based on the above results using the method of "one time one factor", further statistical experimental design methodology was applied to evaluate the interaction and determine the optimal level of three influencing factors including temperature, initial glucose and initial L-phe levels. For the response surface analysis, 17 experiments with triplicates were conducted according to the RSM design, where $\mathrm{Y}$ is 2-PE production $(\mathrm{g} / \mathrm{L}), \mathrm{X} 1$ denotes temperature (), X2 denotes initial glucose level $(\mathrm{g} / \mathrm{L})$ and X3 denotes initial L-phe level $(\mathrm{g} / \mathrm{L})$ (Table 3). According to the response values obtained from the experimental results, a second order regression equation was generated for the response surface, as follows:

$\mathrm{Y}=2.28+0.013 \mathrm{X} 1-0.12 \mathrm{X} 2+0.072 \mathrm{X} 3-0.12 \mathrm{X} 1 \mathrm{X} 2+0.032 \mathrm{X} 1 \mathrm{X} 3+0.0016 \mathrm{X} 2 \mathrm{X} 3-0.34 \mathrm{X} 12-0.87 \mathrm{X} 22-$ $0.50 X 32($ Eq. 2, $[\mathrm{R} 2=0.9710 ; \mathrm{R} 2(\mathrm{Adj})=0.9337 ; \mathrm{R} 2($ Pred $)=0.5362])$. The regression model $($ Eq. $(1))$ gave a high R2 value of 0.9710 (Table 4), which indicated aptness of the model (Isar, Agarwal, Saran, \& Saxena, 2006). The R2 value is between 0 and 1 . When R2 is close to 1.0, the model will better predict the response (Aghaie, Pazouki, Hosseini, Ranjbar, \& Ghavipanjeh, 2009). Notably, the model F value of 26.06 and values of probability $(\mathrm{P})>\mathrm{F}(0.0001)$ indicated that the model terms were significant (Bezerra, Santelli, Oliveira, Villar, \& Escaleira, 2008). The three-dimensional response surface plot is generally used to demonstrate relationships between the response and experimental levels of each variable (Fig. 3). The highest point on the contour profiles in Fig. 3 indicates the optimal parameter values of the highest 2-PE production. As shown, there was obvious interaction between each pair of variables, and the interaction between the selected three variables was significant. The optimal 2-PE production value predicted from the response surface model was $2.37 \mathrm{~g} / \mathrm{L}$, when the temperature was controlled at about 24.7 and initial glucose and L-phe concentrations were 63.5 and $10.7 \mathrm{~g} / \mathrm{L}$, respectively.

The following validation experiment in $3.0 \mathrm{~L}$ bioreactor was carried out under the optimized conditions with $63.54 \mathrm{~g} / \mathrm{L}$ of initial glucose, 10.70 of L-phe $\mathrm{g} / \mathrm{L}$ at 24.7 . The 2-PE production was finally improved up to $2.55 \mathrm{~g} / \mathrm{L}$ after $96 \mathrm{~h}$, which is $7.8 \%$ higher than the predicted level, representing the highest 2-PE production from L-phe by using M. guilliermondii (Karolina et al., 2017). Hence, the models developed were considered to be accurate and reliable for predicting 2-PE production from L-phe by using strain YLG18.

\subsection{Biosynthesis of 2-PE by using Meyerozyma sp. strain YLG18 with ISPR technology}

As mentioned, 2-PE is toxic to microbial cells, as it can make the lipid membrane structure a preferential binding target, resulting in the collapse of transmembrane gradients and the loss of cell viability (Sikkema et al., 1995). To further increase the final 2-PE production and productivity, more robust strains or novel extraction technology should be developed. In situ product recovery (ISPR) techniques can simultaneously remove 2-PE from the fermentation broth while it is produced. Thereby, 2-PE concentration could maintain below the inhibitory level, and microbes are able to continuously produce 2-PE. Suitable extractants including fatty acid methyl ester (FAME), oleic acid and ethyl acetate were first identified. As seen from Fig. 4A, the 
partition coefficients of these organic solvents were similar for 2-PE. However, different organic solvents had different impacts on the dissolved oxygen level, which would affect microbial growth and lead to significant difference in the final 2-PE titer (Weber \& Bont, 1996). Among these organic solvents, FAME gave the highest $2.53 \mathrm{~g} / \mathrm{L}$ of 2-PE. The volume ratio of medium and extractant phases will also affect the distribution and mass transfer of products. Low ratio of FAME could not extract 2-PE effectively, affecting the bioconversion rate. High ratio of FAME may increase toxicity to cells. Results showed that the highest $2.48 \mathrm{~g} / \mathrm{L}$ of 2-PE was obtained when FAME : water ratio was maintained at 1:1 (Fig. 4B). It should be noticed that when the ratio of FAME to water was 1:2, the production of $2-\mathrm{PE}$ was $2.45 \mathrm{~g} / \mathrm{L}$, which is close to the highest one. Accordingly, the optimal ratio of 1:2 was chosen for following experiments.

Generally, microbes would show a log phase after inoculated into the fresh medium. The presence of FAME in the initial stage may prolong this period and affect the transformation activity of yeasts. Therefore, a proper delay of FAME addition may help to improve the metabolic activity of yeasts and ultimately increase 2-PE production. From Fig. 4C, it can be seen that the earlier extractants were added, the more obvious the strain growth inhibition was. Compared with the experimental results of groups 4, 5 and 6 , when FAME was supplemented when strain growth reached exponential phase, the death rate would be decreased significantly. In addition, it can be seen from Fig. 4D that when the strain death rate decreases, the 2-PE production in the group 4 was also the highest, and it can reach $3.20 \mathrm{~g} / \mathrm{L}$, which was improved by $25.49 \%$ compared to single-phase biotransformation.

\subsection{Characterization of related genes and biosynthetic pathway of 2-PE by Meyerozyma sp. strain YLG18 through transcriptome analysis}

At present, only limited literatures have reported 2-PE production by using M. guilliermondii. To further elaborate the 2-PE production mechanism by strain YLG18, genes involved in substrate consumption, L-phe metabolism and 2-PE production were identified and characterized, which could help speculate the 2-PE biosynthesis pathway and provided clues on the divergence of gene expression level between Shikimate pathway and Ehrlich pathway (Fig. 5). After integrating the annotations of strain genome sequences in GO, COG and KEGG, total 10 main unigenes (aroF, aroM, CM, aroC, pheA, AAT, ARO8, HisC, PDC, ADH ) with functions assigned to 2-PE biosynthesis were identified. According to KEGG annotation, the first five genes are key ones in the Shikimate pathway, which were mainly responsible for conversion of glucose into phenylpyruvate; while the last five genes are key ones in the Ehrlich pathway, which were mainly responsible for conversion of L-phe into 2-PE. As for the Shikimate pathway in strain YLG18, a penta-functional enzyme of AroM was identified, which is different from some prokaryotes, whose Shikimate pathway reactions are mainly catalyzed by enzymes encoded by individual genes ( $\operatorname{aro} B$, aroD , aro $E$, aroK, aro $A$ ). To further confirm the function of this penta-functional enzyme AroM, the protein sequence was blasted in NCBI website of non-redundant protein sequences (nr) database. Only 59.33\% similarity was shown between AroM from strain YLG18 and the penta-functional protein ARO1p from S. cerevisiae S288C (NP_010412.1). Previous studies have reported that the penta-functional protein ARO1 in $S$. cerevisiae is essential for aromatic amino acid production (Gold et al., 2015; Hassing et al., 2019). Although AroM in M. guilliermondii complicates tuning gene expression at individual activity level, it can convert 3-deoxy-D-arabinoheptulosonate-7-phosphate (DAHP) to 5-enolpyruvyl-shikimate-3-phosphate (EPSP), which is important in Shikimate pathway. This newly identified penta-functional enzyme AroM from strain YLG18 may broaden our knowledge of 2-PE production through Shikimate de novo pathway.

To confirm the reproducibility and accuracy of differential gene expression identified through the Illumina analysis, genes related to phenylalanine biosynthesis and phenylalanine metabolism were selected for qRTPCR analysis to determine the FPKM value. As can be seen from Fig. 5, the expression levels of genes in Ehrlich pathway were higher than those in Shikimate pathway except for HisC. The overall change in the expression pattern for Ehrlich pathway vs. Shikimate pathway ranged from 0.95-fold to 62.68- fold. This result can effectively explain that the 2-PE production synthesized through Ehrlich pathway was generally higher than that synthesized through Shikimate pathway. It is important to point out that the expression of aspartate aminotransferase (AAT) shows nearly 62 times difference in these two pathways. Although 
substrates were different, the expression of ARO8, which is also an amino acid aminotransferase was far lower than that of AAT. This means that AAT plays an important role in the Ehrlich pathway of strain YLG18. Different from the existing results on 2-PE production by other yeasts such as $S$. cerevisiae, various amino acid transaminases (Aro8, Aro9, Bat1, and Bat2) have shown to catalyze the first step of Ehrlich pathway in the catabolism of aromatic amino acids(Kim, Cho, \& Hahn, 2014; Yin et al., 2015). However, AAT has rarely been reported for 2-PE production. Shrawder et al. (Shrawder \& Martinez-Carrion, 1972) have proved that AAT from porcine heart had phenylalanine transaminase activity, and Cardenas-Fernandez et al. (Cardenas-Fernandez, Lopez, alvaro, \& Lopez-Santin, 2012) have successfully synthesized L-phenylalanine with immobilized AAT. Therefore, AAT may be a potential enzyme for 2-PE synthesis, and Meyerozyma sp. strain YLG18 may also serve as a potential candidate for industrial 2-PE production from L-phe. Future studies are needed to elaborate AroM and AAT function, and the tolerance mechanism of strain YLG18.

\section{Conclusion}

This study presents how a newly identified M. guilliermondiistrain YLG18 can be used as a potential candidate for high 2-PE production from L-phe. After determination of influencing factors, process optimization using RSM and reducing toxicity of 2-PE with ISPR techniques, $3.20 \mathrm{~g} / \mathrm{L}$ of $2-\mathrm{PE}$ can be produced from $63.54 \mathrm{~g} / \mathrm{L}$ of glucose and $10.70 \mathrm{~g} / \mathrm{L}$ of L-phe. The high 2-PE production by using strain YLG18 indicates that it may show great potential for 2-PE production. Candidate genes related to 2-PE biosynthesis and metabolism can be used as target genes for marker-assisted selection through genetic engineering to further improve final 2-PE titer in future studies.

\section{Acknowledgements}

This work was supported by National Key Research and Development Program of China (2018YFA0902200), National Natural Science Foundation of China (21978130, 21706125, 31961133017), Jiangsu Province Natural Science Foundation for Youths (BK20170993), Project of State Key Laboratory of Materials-Oriented Chemical Engineering (ZK201601), and Jiangsu Synergetic Innovation Center for Advanced Bio-Manufacture.

\section{References}

Aghaie, E., Pazouki, M., Hosseini, M. R., Ranjbar, M., \& Ghavipanjeh, F. (2009). Response surface methodology (RSM) analysis of organic acid production for Kaolin beneficiation by Aspergillus niger. Chemical Engineering Journal, 147 (2-3), 245-251.

Azevedo, D. L. L., Santos, D. R. H., Vieira, d. Q. M., Gomes, F. L., \& Batista, d. S. W. (2018). Screening of Yeasts Isolated from Brazilian Environments for the 2-Phenylethanol (2-PE) Production.Biotechnology $\mathcal{E}^{3}$ Bioprocess Engineering, 23 (3), 326-332.

Bezerra, M. A., Santelli, R. E., Oliveira, E. P., Villar, L. S., \& Escaleira, L. A. (2008). Response surface methodology (RSM) as a tool for optimization in analytical chemistry. Talanta, 76 (5), 0-977.

Cardenas-Fernandez, M., Lopez, C., alvaro, G., \& Lopez-Santin, J. (2012). 1-Phenylalanine synthesis catalyzed by immobilized aspartate aminotransferase. Biochemical Engineering Journal, 63 (none), 15-21.

Diniz, R. H. S., Rodrigues, M. Q. R. B., Fietto, L. G., Passos, F. M. L., \& Silveira, W. B. (2013). Optimizing and validating the production of ethanol from cheese whey permeate by Kluyveromyces marxianus UFV3.Biocatalysis and Agricultural Biotechnology, 3 (2), 111-117.

Eshkol, N., Sendovski, M., Bahalul, M., Kashi, Y., \& Fishman, A. (2015). Production of 2-phenylethanol from L-phenylalanine by a stress tolerant Saccharomyces cerevisiae strain. Chemical Engineering Journal, 259 (45), 795-805.

Etschmann, M., Bluemke, W., Sell, D., \& Schrader, J. (2002). Biotechnological production of 2-phenylethanol. Applied Microbiology 83 Biotechnology, 59 (1), 1-8.

Feng, L., Wang, M., Wang, J., Zang, S., Xia, W., \& Sheng, L. (2015). Isolation of 2-phenylethanol biosynthesis related genes and their relationship with 2-phenylethanol accumulation in Rosa rugosa.Acta Physiologiae 
Plantarum, 37 (12), 256.

Ferreira, P. G., da Silveira, F. A., Vieira dos Santos, R. C., Andre Genier, H. L., Santos Diniz, R. H., Ribeiro Junior, J. I., . . . da Silveira, W. B. (2015). Optimizing ethanol production by thermotolerant Kluyveromyces marxianus CCT 7735 in a mixture of sugarcane bagasse and ricotta whey. Food Science and Biotechnology, 24 (4), 1421-1427.

Hua, D., \& Xu, P. (2011). Recent advances in biotechnological production of 2-phenylethanol. BIotechnology Advances, 29 (6), 654-660.

Huang, C. J., Lee, S.-L., \& Chou, C.-C. (2001). Production of 2-phenylethanol, a flavor ingredient, by Pichia fermentans L-5 under various culture conditions. Food Research International, 34 (4), 0-282.

Isar, J., Agarwal, L., Saran, S., \& Saxena, R. K. (2006). A statistical method for enhancing the production of succinic acid from Escherichia coli under anaerobic conditions. Bioresource Technology, 97 (13), $1443-1448$.

Karolina, C., Katarzyna, S. M., Daria, K. P., \& Jolanta, M. (2017). Screening of yeasts for the production of 2-phenylethanol (rose aroma) in organic waste-based media. Letters in Applied Microbiology .

Kim, B., Cho, B.-R., \& Hahn, J.-S. (2014). Metabolic engineering of Saccharomyces cerevisiae for the production of 2-phenylethanol via Ehrlich pathway. Biotechnology and Bioengineering, 111 (1), $115-124$.

Livak, K., \& Schmittgen, T. (2000). Analysis of Relative Gene Expression Data Using Real-Time Quantita-

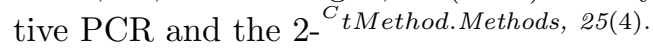

Masuo, S., Osada, L., Zhou, S., Fujita, T., \& Takaya, N. (2015). Aspergillus oryzae pathways that convert phenylalanine into the flavor volatile 2-phenylethanol. Fungal Genetics and Biology, 77, 22-30.

Mu, L., Hu, X., Liu, X., Zhao, Y., \& Xu, Y. (2014). PRODUCTION OF 2-PHENYLETHANOL BY MICROBIAL MIXED CULTURES ALLOWS RESOURCE RECOVERY OF CANE MOLASSES WASTEWATER. Fresenius Environmental Bulletin, 23 (6), 1356-1365.

Papon, N., Savini, V., Lanoue, A., Simkin, A. J., Creche, J. l., Giglioli-Guivarca'h, N., Clastre, M., Courdavault, V., Sibirny, A. A. (2013). Candida guilliermondii: biotechnological applications, perspectives for biological control, emerging clinical importance and recent advances in genetics. Current Genetics, 59 (3), 73-90.

Perdiguero, P., Collada, C., Barbero, M. d. C., Casado, G. G., Cervera, M. T., \& Soto, a. (2012). Identification of water stress genes in Pinus pinaster Ait. by controlled progressive stress and suppression-subtractive hybridization. Plant Physiology and Biochemistry, 50 (none), 44-53.

Schrader, J., Etschmann, M. M. W., Sell, D., Hilmer, J. M., \& Rabenhorst, J. (2004). Applied biocatalysis for the synthesis of natural flavour compounds - Current industrial processes and future prospects.Biotechnology Letters, 26 (6), 463-472.

Scognamiglio, J., Jones, L., Letizia, C. S., \& Api, A. M. (2012). Fragrance material review on phenylethyl alcohol. Food and Chemical Toxicology, 50 (supp_S2), S224-S239.

Seward, R., Willetts, J. C., Dinsdale, M. G., \& Lloyd, D. (1996). THE EFFECTS OF ETHANOL, HEXAN1-OL, AND 2-PHENYLETHANOL ON CIDER YEAST GROWTH, VIABILITY, AND ENERGY STATUS; SYNERGISTIC INHIBITION. Journal of the Institute of Brewing, 102 (6), 439-443.

Shen, L., Nishimura, Y., Matsuda, F., Ishii, J., \& Kondo, A. (2016). Overexpressing enzymes of the Ehrlich pathway and deleting genes of the competing pathway in Saccharomyces cerevisiae for increasing 2-phenylethanol production from glucose. Journal of Bioscience and Bioengineering, 122 (1), 34-39.

Shrawder, E., \& Martinez-Carrion, M. (1972). Evidence of phenylalanine transaminase activity in the isoenzymes of aspartate transaminase. Journal of Biological Chemistry, 247 (8), 2486-2492. 
Sikkema, J., de Bont, J. a., \& Poolman, B. (1995). Mechanisms of membrane toxicity of hydrocarbons. Microbiological reviews, 59 (2), 201-222.

Stark, D. (2003). In situ product removal (ISPR) in whole cell biotechnology during the last twenty years. Advances in Biochemical Engineering Biotechnology, 80 .

Stark, D., Munch, T., Sonnleitner, B., Marison, I. W., \& Stockar, U. V. (2002). Extractive Bioconversion of 2-Phenylethanol from L-Phenylalanine by Saccharomyces cerevisiae. Biotechnology Progress, 18 (3), 514-523.

Stark, D., Zala, D., Munch, T., Sonnleitner, B., Marison, I. W., \& Stockar, U. v. (2003). Inhibition aspects of the bioconversion of 1-phenylalanine to 2-phenylethanol by Saccharomyces cerevisiae.Enzyme and Microbial Technology, 32 (2), 212-223.

Weber, F. J., \& Bont, J. A. M. d. (1996). Adaptation mechanisms of microorganisms to the toxic effects of organic solvents on membranes.Biochimica et Biophysica Acta, 1286 (3), 225-245.

Yin, S., Zhou, H., Xiao, X., Lang, T., Liang, J., \& Wang, C. (2015). Improving 2-Phenylethanol Production via Ehrlich Pathway Using Genetic EngineeredSaccharomyces cerevisiaeStrains. Current Microbiology, 70 (5), 762-767.

\section{Figure Legends}

Fig. 1: 2-PE production and conversion yield byusing different screened strains (A), Growth and 2-PE production of strain YLG18 under two synthetic pathways (B), Tolerance of strain YLG18 to exogenous 2-PE (C).

Fig. 2: Optimization of single factor fermentation conditions: carbon sources (A), fermentation temperatures (B), initial glucose concentrations (C), initial L-phe concentrations (D).

Fig. 3: Three dimensional response surface and contour plot showing the effect of temperature, initial glucose concentration and initial L-phe concentration on 2-PE production $(\mathrm{g} / \mathrm{L})$ : the effect of temperature and initial glucose concentration on 2-PE production $(\mathrm{g} / \mathrm{L})(\mathrm{A})$, the effect of temperature and initial L-phe concentration on 2-PE production (g/L) (B), the effect of initial glucose concentration and initial L-phe concentration on 2-PE production $(\mathrm{g} / \mathrm{L})(\mathrm{C})$, growth and fermentation profiles of strain YLG18 in mineral salts medium amended with $63.54 \mathrm{~g} / \mathrm{L}$ of glucose and $10.70 \mathrm{~g} / \mathrm{L}$ of L-phe with temperature controlled at 24.7 (D).

Fig. 4: Biosynthesis of 2-PE with ISPR technology: 2-PE yield using different extractants (A); 2-PE production with different phase-volume ratios (B); Effects of adding time of FAME on the growth of cells (C); Effects of FAME adding time on 2-PE production(D).

Fig. 5: Proposed metabolic pathway for 2-PE production within strain YLG18 and comparison of expression levels of related genes in two different 2-PE synthesis pathways.

Table 1: Primers used for qRT-PCR analysis.

\begin{tabular}{|c|c|c|c|c|}
\hline KO ID & $\begin{array}{l}\text { Predicted } \\
\text { function }\end{array}$ & Gene name & $\begin{array}{l}\text { Primers used for } \\
\mathrm{qPCR}\end{array}$ & $\begin{array}{l}\text { Primers used for } \\
\mathrm{qPCR}\end{array}$ \\
\hline K13830 & $\begin{array}{l}\text { pentafunctional } \\
\text { AROM polypeptide }\end{array}$ & aroM & F R & $\begin{array}{l}\text { GCGGAATCGAACATTTGTC } \\
\text { TTGAGGGTTTTCAGCGGC }\end{array}$ \\
\hline K01736 & chorismate synthase & aroC & F R & $\begin{array}{l}\text { ATTCAGAGTGACCACCTAT } \\
\text { CACAAGCATACCAATTGGA }\end{array}$ \\
\hline K01626 & $\begin{array}{l}\text { 3-deoxy-7- } \\
\text { phosphoheptulonate } \\
\text { synthase }\end{array}$ & $\operatorname{aroF}$ & F R & $\begin{array}{l}\text { ATACGATGACACACGTAT } \\
\text { CGTACTCTATAGCAGACG }\end{array}$ \\
\hline
\end{tabular}




\begin{tabular}{|c|c|c|c|c|}
\hline KO ID & $\begin{array}{l}\text { Predicted } \\
\text { function }\end{array}$ & Gene name & $\begin{array}{l}\text { Primers used for } \\
\text { qPCR }\end{array}$ & $\begin{array}{l}\text { Primers used for } \\
\text { qPCR }\end{array}$ \\
\hline K03786 & $\begin{array}{l}\text { 3-dehydroquinate } \\
\text { dehydratase }\end{array}$ & aroQ & F R & $\begin{array}{l}\text { CAACCTTTTGGGTACCAGA } \\
\text { TGGCCACAGAAGTATGAGI }\end{array}$ \\
\hline K01850 & chorismate mutase & $\mathrm{CM}$ & F R & $\begin{array}{l}\text { AACCAGAAACTGTTCTTGA } \\
\text { GCCTCGTATCTACGGATCT }\end{array}$ \\
\hline K00817 & $\begin{array}{l}\text { histidinol-phosphate } \\
\text { aminotransferase }\end{array}$ & HisC & F R & $\begin{array}{l}\text { GTGAACCTGCGGAAGGAT } \\
\text { TCATCGATGCGAGAACCA }\end{array}$ \\
\hline K04518 & $\begin{array}{l}\text { prephenate } \\
\text { dehydratase }\end{array}$ & pheA & F R & $\begin{array}{l}\text { TCCAGGCACATATACTCAT } \\
\text { TGTTCTCCCACAATTCTAA }\end{array}$ \\
\hline K14454 & $\begin{array}{l}\text { aspartate } \\
\text { aminotransferase }\end{array}$ & AAT & F R & $\begin{array}{l}\text { CGTGTCCTGTGGATCCAG } \\
\text { TTCTAGCTCCTTCAATGTA }\end{array}$ \\
\hline K00838 & $\begin{array}{l}\text { aromatic amino acid } \\
\text { aminotransferase }\end{array}$ & $\mathrm{ARO} 8$ & $\mathrm{~F} \mathrm{R}$ & $\begin{array}{l}\text { CTTCTATCTGAACAAGCCA } \\
\text { GTCGGTTGAATCCTGCGA }\end{array}$ \\
\hline K12732 & $\begin{array}{l}\text { phenylpyruvate } \\
\text { decarboxylase }\end{array}$ & $\mathrm{PDC}$ & F R & $\begin{array}{l}\text { GGTGAAAGCAGTAGCCAAC } \\
\text { TTGTCTCGATTGACGAGG }\end{array}$ \\
\hline K00002 & $\begin{array}{l}\text { alcohol } \\
\text { dehydrogenase }\end{array}$ & $\mathrm{ADH}$ & F R & $\begin{array}{l}\text { CAAGGTTTTGGAGTCGACC } \\
\text { AGCGTAACTTTGTCACCAA }\end{array}$ \\
\hline
\end{tabular}

Table 2: Top matches of 18S rRNA gene sequence of strain YLG18 against known strains.

\begin{tabular}{ll}
\hline Microorganisms & Identity (\%) \\
\hline Meyerozyma guilliermondii culture CBS:2030 internal transcribed spacer 1, partial sequence & 99 \\
Pichia guilliermondii strain ATCC6260; ATCC46036 internal transcribed spacer 1, partial sequence & 99 \\
Candida athensensis strain BG02-5-23-003I-4 18S ribosomal RNA gene, partial sequence & 95 \\
Carpophila culture CBS:5256 internal transcribed spacer 1, partial sequence & 98 \\
Meyerozyma caribbica CBS 9966 ITS region; from TYPE material & 98 \\
Meyerozyma amylolytica strain DSM 27310 small subunit ribosomal RNA gene, partial sequence & 93 \\
\hline
\end{tabular}

Table 3: RSM experimental design for 2-PE production.

\begin{tabular}{lllll}
\hline Run & X1 & X2 & X3 & Y \\
\hline 1 & 30 & 60 & 5 & 1.56 \\
2 & 25 & 30 & 15 & 1.54 \\
3 & 20 & 60 & 15 & 2.06 \\
4 & 25 & 60 & 10 & 2.35 \\
5 & 25 & 90 & 5 & 1.60 \\
6 & 30 & 30 & 10 & 1.29 \\
7 & 30 & 90 & 10 & 1.99 \\
8 & 25 & 60 & 10 & 2.35 \\
9 & 20 & 90 & 10 & 1.55 \\
10 & 25 & 60 & 10 & 2.35 \\
11 & 25 & 90 & 15 & 1.66 \\
12 & 20 & 60 & 5 & 1.60 \\
13 & 30 & 60 & 15 & 1.66 \\
14 & 25 & 30 & 5 & 1.40
\end{tabular}




\begin{tabular}{lllll}
\hline Run & X1 & X2 & X3 & Y \\
\hline 15 & 25 & 60 & 10 & 2.35 \\
16 & 25 & 60 & 10 & 2.35 \\
17 & 20 & 30 & 10 & 1.61 \\
\hline
\end{tabular}

Table 4: Analysis of variance (ANOVA) for the quadratic model (Coefficient of determination $(\mathrm{R} 2)=0.9710 ;$ Adjusted R2 $=0.9337 ;)$.

\begin{tabular}{lllllll}
\hline Source & Sum of Squares & df & Mean Square & F Value & Prob¿F & Significance \\
\hline Model & 2.28 & 9 & 0.25 & 26.06 & 0.0001 & significant \\
Residual & 0.068 & 7 & 0.009743 & & & \\
Lack of Fit & 0.068 & 3 & 0.023 & & & \\
Pure Error & 0.000 & 4 & 0.000 & & & \\
Cor Total & 2.35 & 16 & & & & \\
\hline
\end{tabular}

\section{Hosted file}

Figures_11.2019.pdf available at https://authorea.com/users/285586/articles/409264-enhanced2-phenylethanol-production-by-newly-isolated 